\title{
Quantification of Event-Related Desynchronization/Synchronization at Low Frequencies in a Semantic Memory Task
}

\author{
Juan Gómez, Mónica Aguilar, Eduardo Horna and Javier Minguez
}

\begin{abstract}
Although several techniques have been developed for the visualization of EEG event-related desynchronization/synchronization (ERD/ERS) in both time and frequency domains, none of the quantification methods takes advantage of the time and frequency resolution at the same time. Existing techniques for the quantification of the ERD/ERS changes compute the average EEG power increase/decrease relative to certain reference value, over fixed time intervals and/or frequency bands (either fixed or individualized). Inaccuracy in the computation of these frequency bands (where the process is actually measured) in combination with the averaging process over time may lead to errors in the computation of any ERD/ERS quantification parameter. In this paper, we present a novel method for the automatic, individual and exact quantification of the most significant ERD/ERS region within a given window of the time-frequency domain. The method is exemplified by quantifying the ERS at low frequencies of 10 subjects performing a semantic memory task, and compared with existing techniques.
\end{abstract}

\section{INTRODUCTION}

The correlation between brain rhythms and certain brain functions has been widely demonstrated by time-frequency analysis of the EEG. A common practice when analyzing EEG power changes induced by certain mental processes is the calculation of event-related desynchronization or synchronization (ERD/ERS) within a given time-frequency window. This technique has been used in many contexts such as the study of alpha and theta power changes induced by attentional and semantic memory demands [1], [2], the relation between upper-alpha power and memory performance and intelligence [3], [4], or theta power changes during online sentence processing [5].

The time-frequency window of interest is usually defined by a fixed time interval (given by the experimentation protocol) and the frequency band of interest (given by neurophysiological basis). Beforehand fixed frequency bands with fixed widths (FBFW) have been traditionally used, ranging from 0.5 to $4 \mathrm{~Hz}$ (delta), 4-7 Hz (theta), 7-13 Hz (alpha), 13-30 (beta) and frequencies faster than $30 \mathrm{~Hz}$ (gamma). However, fixed frequency bands show large interindividual differences due to several factors such as age, memory performance, brain volume or task demands [6], [7]. Therefore, several

Juan Gómez was with the University of Zaragoza and is with BitBrain Technologies S.L., juan.gomez.cadena@gmail.com. Mónica Aguilar is with BitBrain Technologies S.L., Zaragoza, Spain, monicaaguilarabitbrain.es. Eduardo Horna is with the University of Zaragoza (I3A), eduardo . horna @ gmai 1 . Com. Javier Minguez is with the University of Zaragoza (I3A) and BitBrain Technologies S.L., Spain, jminguez@unizar.es. This work was partially supported by the INNCORPORA program of the Spanish Ministry of Science and Innovation, by Spanish projects HYPER-CSD2009-00067 and DPI2009-14732-C02-01, and by DGA-FSE (T04 group).

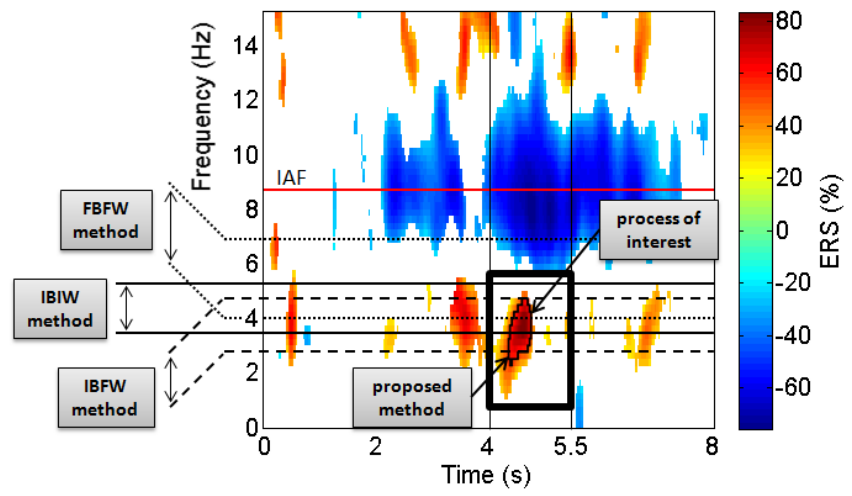

Fig. 1. ERD/ERS time-frequency map of the task. The black rectangle points the ERS of interest (traditionally associated with the theta band) as reflecting the semantic memory and encoding demands. The resulting theta band computed by the three classic methods FBFW, IBFW and IBIW is depicted by three separate pairs of bold and dashed black lines, while for the proposed method, the contour of the resulting most significant ERS region is depicted. Notice how the FBFW and the IBIW methods failed to capture the main synchronization process, while the IBFW achieved a very well fitted frequency band in this case. With regard to the temporal domain, none of the three classic methods captured the synchronization process in time as the time window is fixed beforehand.

techniques have emerged to adjust the frequency bands individually for each subject [6], [8]. In this context, alpha and theta are frequently determined for each subject according to the individual alpha frequency (IAF), a physiological anchor point traditionally defined as the frequency of the spectra that carries more power within alpha band. Calculation of individual bands with either fixed (IBFW) or individual widths (IBIW) [8] are the most accepted methods for frequency bands individualization. The first one computes alpha band as [IAF-4, IAF+2] Hz and theta as [IAF-6, IAF-4] Hz. The second method adjusts the frequency bands in terms of a certain percentage of the IAF (i.e. $20 \%$ was suggested) to avoid misalignment of slower frequencies.

Once the time and frequency bounds have been determined, ERD/ERS values are usually computed within the window of interest as the average increase or decrease (in percentage terms) of the EEG power relative to certain reference interval over time, frequency or both [1], [8], [9]. Inaccuracy in the computation of these frequency bands (where the process is actually measured) in combination with the averaging process over time may lead to errors in the computation of any ERD/ERS quantification parameter. Fig. 1 exemplifies this problem.

The present paper describes a novel method for the automatic, individual and exact quantification of the most 
statistically significant ERD/ERS region in a given window of the time-frequency domain (see the contour of the resulting region computed by the proposed method in Fig. 1). In order to validate this technique, a semantic memory task that involves a synchronization in the low frequencies of the EEG spectra (traditionally located in the theta band) was executed [1]. To the best of the author's knowledge, there are no techniques for both the exact identification of the ERD/ERS and its quantification in a given time-frequency window. For this reason, the performance of the proposed technique has been compared with the three classic methods already mentioned (FBFW, IBFW and IBIW) to compute the theta band in the frequency domain. The results demonstrate how the new technique identifies and quantifies with high accuracy the ERS in the low frequencies.

\section{METHODS}

\section{A. Participants}

10 healthy subjects participated in the study ( 8 males and 2 females in the range $23.7 \pm 0.94$ years). Participants were duly informed about the entire protocol of the study before signing the consent forms.

\section{B. Experimental procedure}

In the experiment (replicated from [1]), the subjects had to judge whether sequentially presented feature-concept pairs of words were semantically congruent, responding "yes" to a congruent pair (e.g. 'black-raven') and "no" to an incongruent pair (e.g. 'huge-pea'). A warning signal preceded the presentation of the "feature" word. After the "concept" word, subjects give an answer as quickly as possible after a cue signal. The experimental design is shown in Fig. 2.

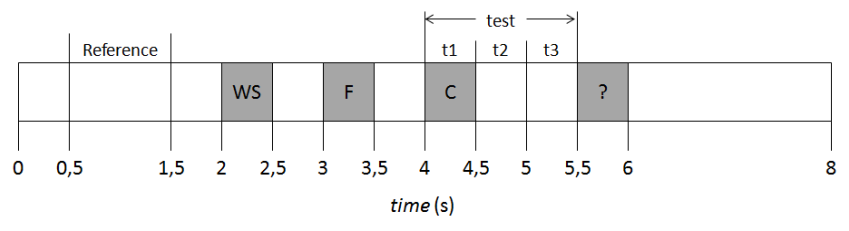

Fig. 2. Structure of a single trial. "Feature" (F) and "Concept" (C) words are presented in seconds 3 and 4 respectively. The warning signal (WS) is shown one second before the "Feature" word presentation, and the answer is requested 1.5 seconds after "Concept" word is presented. All events have a duration of $500 \mathrm{~ms}$. A "Reference" interval was defined to compute ERD/ERS changes along the trial. The test interval (from second 4 to 5.5) comprises the time period within which the studied process is expected to occur. For FBFW, IBFW and IBIW methods, this test interval was divided into three subintervals $\mathrm{t} 1, \mathrm{t} 2$ and $\mathrm{t} 3$, of $500 \mathrm{~ms}$ each, in order study which of them capture the synchronization process better.

\section{EEG Signal recording}

EEG signals were recorded from 16 active electrodes placed at FP1, FP2, F3, Fz, F4, C3, Cz, C4, P7, P3, Pz, P4, P8, O1, Oz and O2 (according to the 10/10 system). Ground and reference electrodes were placed on $\mathrm{FPz}$ and the earlobe, respectively. Signal recordings were amplified using a commercial gTec system at a sampling rate of $256 \mathrm{~Hz}$, and bandpass filtered between 0.5 and $30 \mathrm{~Hz}$. Signal acquisition and processing were developed using a self-made software coded in $\mathrm{C}++$ and running on a Windows computer.

\section{EEG Signal processing}

Blinking artifact filtering was performed using the FastICA algorithm [10] and a $100 \mu \mathrm{V}$ threshold to discard the artifacted independent components. EEG power spectrum was calculated by means of a sliding window FFT algorithm: a 128 samples Hamming window with 120 samples of overlapping. Resolution was set to $0.25 \mathrm{~Hz}$ (1024 points using zero-padding). The IAF was computed for each subject as the peak frequency (PF) within the [6.5 - 13] $\mathrm{Hz}$ band. The ERD/ERS time-frequency maps showing only significant values were computed using the bootstrap-based method reported in [11], [12], where the ERD/ERS calculation is performed relative to a certain reference interval $(\alpha=0.05)$. As in the original experiment [1], we established a reference interval from $500 \mathrm{~ms}$ to $1500 \mathrm{~ms}$ (see Fig. 2).

\section{E. Identification and quantification of ERD/ERS}

In the present study, the three methods assessed in [8] for frequency bands determination were implemented and compared with the proposed method:

1) Existing methods: The three classic methods are: i) Fixed bands and fixed widths (FBFW), where theta ranges from 4 to $6 \mathrm{~Hz}$; $i$ ) Individually defined bands and fixed widths (IBFW), where theta ranges from (IAF-6) to (IAF-4) Hz; iii) Individually defined bands and widths (IBIW), where theta width is computed as a percentage of the IAF (20\% is suggested), and ranges from (0.4*IAF) to (0.6*IAF). For these three methods, the test interval was divided into three subintervals of 0.5 second each (t1, t2 and $\mathrm{t} 3$ ) as depicted in Fig. 2, since it is a common practice in the literature.

2) The Most Significant Region method (MSR): The determination of the most significant ERS region of interest is performed from a time-frequency map by the following segmentation algorithm (see Fig.3):

1) Define a time-frequency window of interest: specify the time and frequency bounds within which the studied process is expected to take place. These bounds determine the search area where the algorithm will look for the most significant ERD/ERS values.

2) Find a seed: the starting point for the algorithm is determined by the local maximum of greater ERD or ERS magnitude within the search area (for the sake of simplicity the algorithm is described for ERS detection). Local maxima are computed as points greater than zero whose eight neighbours are lower in ERS magnitude than themselves.

3) Map segmentation: determine all non-zero points connected to the seed by means of a region growing algorithm. In this step, the minimum ERS value within the resulting region is also computed. 
4) Reduction: optionally, a reduction of the region determined in step 3 can be performed to keep only the most representative subregion. This reduction is computed establishing a threshold for the region growing algorithm, calculated as follows:

$$
\text { threshold }=E R S_{\text {min }}+k\left(E R S_{\text {seed }}-E R S_{\text {min }}\right)
$$

where $E R S_{\min }$ is the minimum ERS value within the region, $E R S_{\text {seed }}$ is the ERS value at the seed point and $k$ is a constant between 0 and 1 that controls the reduction percentage. Then, the segmentation described in step 3 is performed again using the new threshold as the connectivity cut off value.

The result of this process is the most significant ERS region within the time-frequency window of interest. Then, for each subject, the following metrics for the ERS quantification are computed:

- Frequency interval (FI): determination of the upper and lowermost points of the region, which determine the frequency range where the main ERS is located (Fig. 3).

- Time interval (TI): determination of the left and rightmost points of the resulting region, which determine the time period where the main synchronization takes place (see Fig. 3).

- Mean ERS: calculation of the average ERS within the computed region.

- Maximum ERS: the greatest ERS value within the region computed by the method.

- Total ERS: the sum of all ERS values within the resulting region.

\section{F. Evaluation of the method}

For the assessment of the proposed method, the time period between the presentation of the "concept" word and the response signal was selected as test interval (see Fig. 2). This time interval was chosen because the interest is to study the synchronization at lower frequencies, traditionally associated to the frontal theta band activity, which takes place due to semantic memory and encoding demands [1], [2], [6]. Therefore, left and right bounds for the time-frequency window of interest correspond to the seconds 4 and 5.5 respectively, and the IAF is used here as the upper bound, since the objective is the quantification of the ERS at low frequencies. No lower bound was specified ( $0 \mathrm{~Hz}$ was used). In the present study, an initial reduction using $k=0.5$ was applied, and then $k$ was incremented step by step until the bandwidth was narrower than $3 \mathrm{~Hz}$ (if necessary). Thus, we restricted the maximum height of resulting most significant region to $3 \mathrm{~Hz}$.

\section{RESULTS}

For subjects 1-5, Fig. 4 shows the time-frequency maps computed at Fz location, showing only significant ERD/ERS values. Due to space limitations, subjects 6-10 were not included in the figure. For the MSR method, two horizontal and two vertical bold black lines delimit the most significant

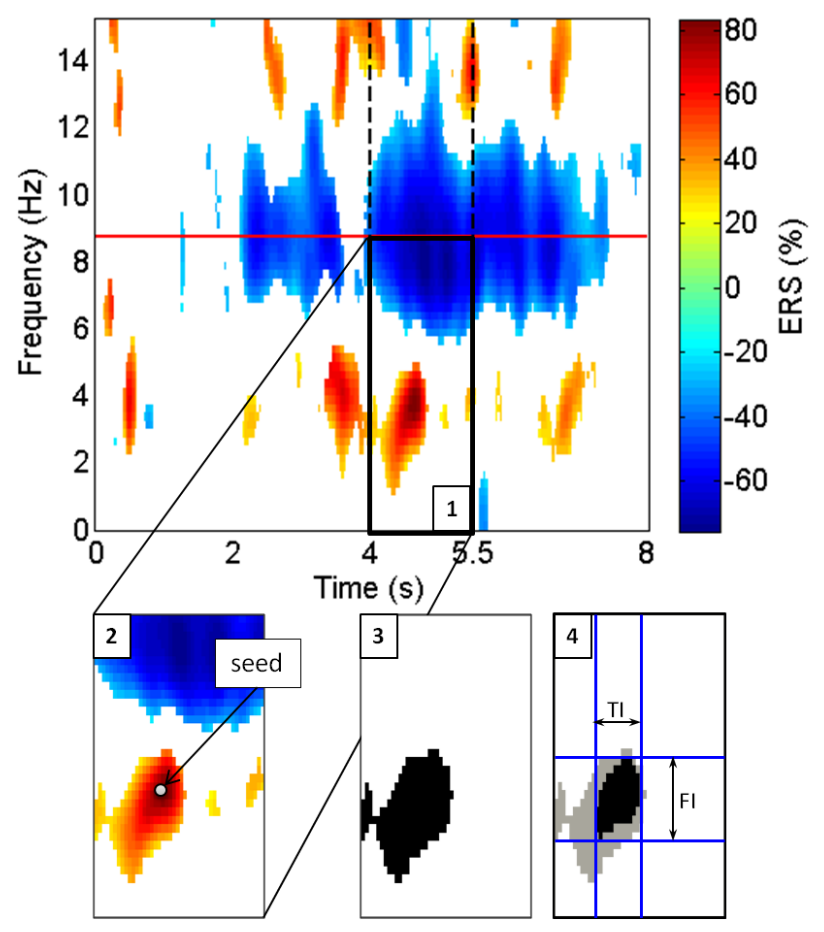

Fig. 3. Graphical representation of the proposed method. On the top, a timefrequency map showing only significant changes of ERD/ERS is presented. IAF is depicted by a horizontal red line, and the search area, defined in step 1 , corresponds to the black rectangle. On the bottom, steps 2 (search of the seed), 3 (segmentation) and 4 (reduction) are depicted. The resulting region after step 4 determines the frequency and time intervals (FI and TI respectively) corresponding to the most significant ERS area.

ERS region within the time-frequency window of interest. For all five subjects, the proposed method succeeded to capture accurately the region where the main synchronization takes place. Subject 3 shows two significant ERS regions, but only the leftmost one is considered, because although the rightmost one contains higher ERS values, choosing the seed by computing local maxima, as explained in the previous section (step 2 of the MSR method), avoids selecting regions whose ERS values are constantly growing as we move towards $0 \mathrm{~Hz}$, and thus corresponding to delta frequencies, which are not associated to the semantic memory process studied. For subject 4, the MSR method captured almost the whole main synchronization, but in this case the semantic memory process extended beyond the 5.5 seconds and merged with another kind of process related to the answer event, so that a small ERS region fell outside the test interval and thus it could not be captured in its entirety. This issue could be addressed by considering a wider time-frequency window.

With regard to the classic methods (FBFW, IBFW and IBIW), Fig. 4 illustrates the negative effects of the association of the synchronization process with the theta band computed according to the IAF. For these methods, two horizontal bold black lines depict the resulting theta band, and the three test subintervals ( $\mathrm{t} 1, \mathrm{t} 2$ and $\mathrm{t} 3$ ) are depicted by vertical dashed lines. For subjects 1 and 5, the FBFW 

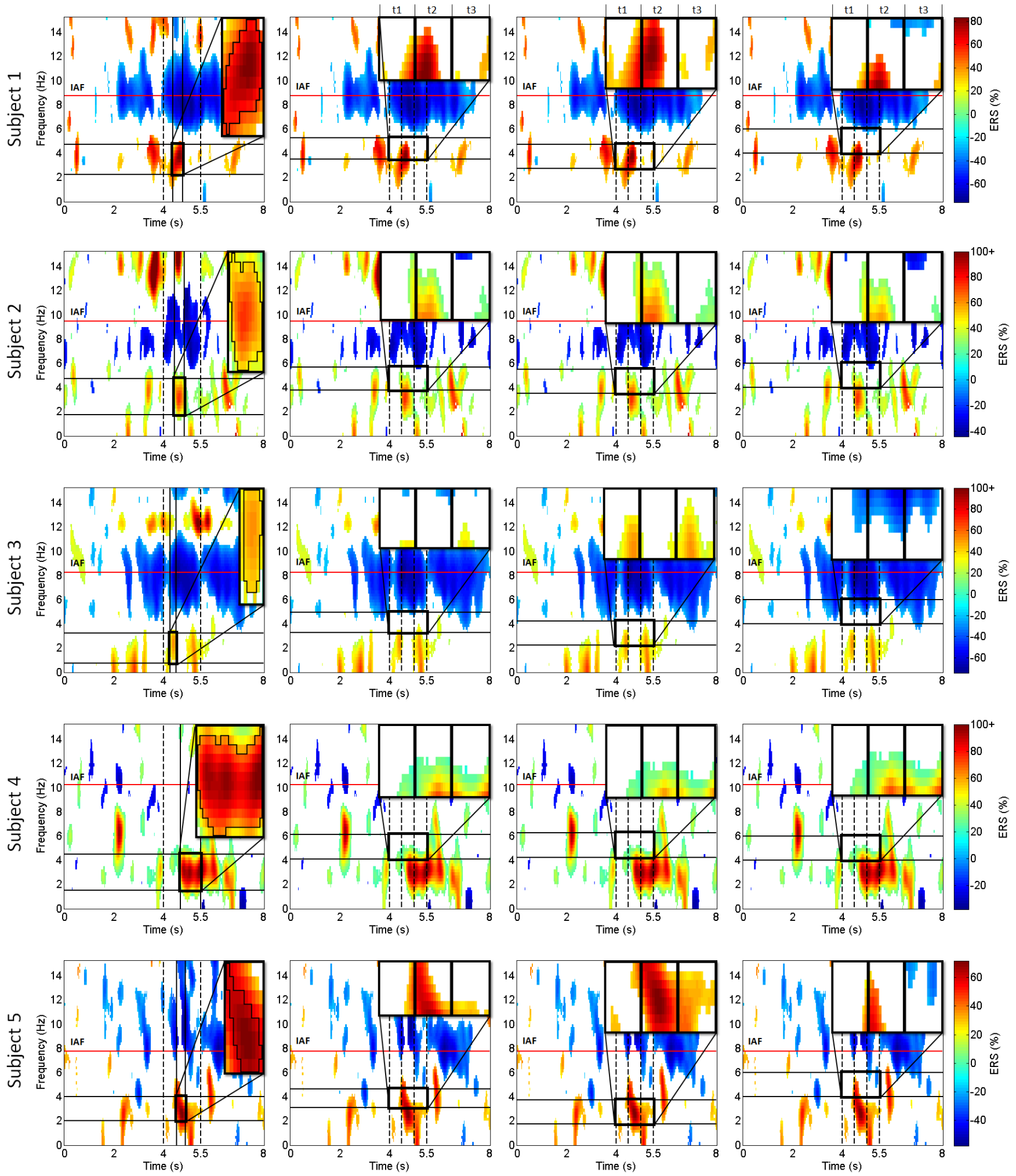

Fig. 4. The figure above shows the time-frequency maps, showing only significant ERD/ERS values, computed at Fz location for subjects 1-5. Two vertical dashed lines depict the test interval (see Fig. 2), and for the three classic methods, two additional lines divide this interval into three subintervals of $500 \mathrm{~ms}$ each, as described in section II-E.1. For the MSR method, the most significant region bounds are depicted by two horizontal and two vertical bold black lines, and the contour of such region is depicted in the zoomed area. For the FBFW, IBFW and IBIW methods, the resulting theta band is depicted by two horizontal bold black lines, and the zoomed area shows the ERD/ERS within intervals $\mathrm{t} 1$, $\mathrm{t} 2$ and $\mathrm{t} 3$ in detail. The IAF for each subject is represented by a horizontal red line. 
method failed to capture the frequency interval where the main synchronization takes place, comprising always higher frequency ranges. In contrast, the IBFW method achieved a very well fitted frequency band when $\mathrm{t} 2$ interval is considered. The IBIW method provided intermediate results, capturing only about a half of the main ERS region. For subject 2 , the three methods worked similarly, determining frequency intervals too high to capture the synchronization process, which only occurred during t 2 . The opposite situation was found for subject 3, which presents significant synchronization in $\mathrm{t} 1$ and $\mathrm{t} 3$, but not in $\mathrm{t} 2$. For this subject only the IBFW method achieved to capture about a half of the main ERS, while the FBFW and IBIW methods failed completely in their objective. Finally, all the classic methods determined too high frequency intervals for subject 4 , and the entire synchronization process lies outside the timefrequency window defined by either $\mathrm{t} 1, \mathrm{t} 2$ or $\mathrm{t} 3$ intervals.

Once the resulting region calculated by the MSR method has been obtained, metrics described in section II-E.2 were computed. Table I shows, for all subjects, the frequency and time intervals (FI and TI respectively) which delimit the resulting region, as well as the average, total and maximum ERS values within such region. In addition to the FI individualization, it is important to stress the capability of the proposed method to capture the time interval (TI) when the synchronization process occurs, since this parameter is fixed for all subjects in the classic methods (set beforehand relative to the experimentation protocol). Moreover, the average, total and maximum values of the synchronization related to the semantic process are computed with high accuracy as they are calculated within the resulting region and not within a rectangular area defined by a time-frequency window. Finally, for subject 10 no metrics are reported as no significant ERS was obtained in the time-frequency window analyzed. Note that this lack of synchronization would not be reported by the classic techniques that will compute the ERS over a time-frequency window (even though there is no significant ERS).

\section{Discussion}

This study highlight the problems associated to the use of individualized bands computed according to the IAF and the use of fixed time windows to quantify ERD/ERS. To address these issues, we proposed a novel method, which shares certain similarities with a recently developed technique [13], capable of automatically determining the most significant ERS region within a window of interest in both time and frequency domains accurately. To exemplify the proposed method, we customized the algorithm to detect ERS at low frequencies, induced by a semantic memory and encoding process traditionally associated to the theta band. This is a specific application of the method, but not the only one. We suggest the customization of the main algorithm to deal with different situations. For example, the seed selection criteria could be changed to compute local minima, and thus the algorithm would determine the most significant ERD region. In addition, the reduction criteria and the time-frequency
TABLE I

The following table shows the frequency (FI) and time (TI) intervals that delimit the resulting most significant region computed by the MSR method for all subjects. In addition, the average, total and maximum ERS within the resulting region was computed. Note that subject 10 had no significant synchronization within the time-frequency window of interest and thus the identification of any relevant ERS region was not possible.

\begin{tabular}{cccccc}
\hline \multirow{2}{*}{ Subject } & \multirow{2}{*}{ FI (Hz) } & \multirow{2}{*}{ TI (s) } & \multicolumn{3}{c}{ ERS $(\%)$} \\
\cline { 4 - 6 } & & & Average & Total $\left(\times 10^{3}\right)$ & Max \\
\hline 1 & {$[2.25,4.75]$} & {$[4.40,4.76]$} & $67.89 \pm 8.20$ & 5.23 & 83.32 \\
2 & {$[1.75,4.75]$} & {$[4.46,4.83]$} & $59.10 \pm 8.05$ & 6.32 & 74.6 \\
3 & {$[0.75,3.25]$} & {$[4.30,4.50]$} & $44.97 \pm 4.35$ & 2.25 & 52.99 \\
4 & {$[1.50,4.50]$} & {$[4.70,5.50]$} & $81.55 \pm 10.98$ & 19.49 & 103.62 \\
5 & {$[2.00,4.00]$} & {$[4.57,4.86]$} & $65.34 \pm 3.55$ & 2.55 & 71.78 \\
6 & {$[4.00,5.00]$} & {$[4.56,4.63]$} & $32.18 \pm 1.49$ & 0.26 & 34.29 \\
7 & {$[3.25,6.25]$} & {$[4.36,4.76]$} & $65.75 \pm 9.31$ & 7.23 & 83.14 \\
8 & {$[2.00,4.25]$} & {$[4.30,4.46]$} & $30.46 \pm 2.26$ & 1.31 & 34.87 \\
9 & {$[2.00,4.25]$} & {$[4.37,4.50]$} & $63.51 \pm 3.68$ & 1.97 & 70.13 \\
10 & - & - & - & - & - \\
\hline
\end{tabular}

window bounds should be adjusted according to the specific needs of the application.

\section{REFERENCES}

[1] W. Klimesch, M. Doppelmayr, T. Pachinger, and H. Russegger. Eventrelated desynchronization in the alpha band and the processing of semantic information. Brain Research, 6(2):83-94, 1997.

[2] W. Klimesch, M. Doppelmayr, T. Pachinger, and B. Ripper. Brain oscillations and human memory: EEG correlates in the upper alpha and theta band. Neuroscience Letters, 238(1-2):9-12, 1997.

[3] M. Doppelmayr, W. Klimesch, K. Hödlmoser, P. Sauseng, and W. Gruber. Intelligence related upper alpha desynchronization in a semantic memory task. Brain Research Bulletin, 66(2):171-177, 2005.

[4] W. Klimesch, M. Doppelmayr, and S. Hanslmayr. Upper alpha ERD and absolute power: their meaning for memory performance. Progress in Brain Research, 159:151-165, 2006.

[5] M. C. M. Bastiaansen, J. J. A. Van Berkum, and P. Hagoort. Eventrelated theta power increases in the human EEG during online sentence processing. Neuroscience Letters, 323(1):13-16, 2002.

[6] W. Klimesch. EEG alpha and theta oscillations reflect cognitive and memory performance: a review and analysis. Brain Res. Reviews, 1999.

[7] Monica Aguilar, Marco Congedo, and Javier Minguez. A datadriven process for the development of an eyes-closed EEG normative database. 2011 Annual International Conference of the IEEE Engineering in Medicine and Biology Society, 2011:7306-7309.

[8] M Doppelmayr, W Klimesch, T Pachinger, and B Ripper. Individual differences in brain dynamics: important implications for the calculation of event-related band power. Biological Cybernetics, 79(1):49-57, 1998.

[9] W. Klimesch, M. Doppelmayr, H. Russegger, T. Pachinger, and J. Schwaiger. Induced alpha band power changes in the human EEG and attention. Neuroscience Letters, 244(2):73-76, 1998.

[10] Aapo Hyvärinen. Fast and robust fixed-point algorithms for independent component analysis. IEEE Trans. on Neural Networks, 1999.

[11] B. Graimann and G. Pfurtscheller. Quantification and visualization of event-related changes in oscillatory brain activity in the time-frequency domain. Progress in Brain Research, 159:79-97, 2006.

[12] B. Graimann, J. E. Huggins, S. P. Levine, and G. Pfurtscheller. Visualization of significant ERD/ERS patterns in multichannel EEG and ECoG data. Clinical Neurophysiology, 113(1):43-47, 2002.

[13] M. Billinger, V. Kaiser, C. Neuper, and C. Brunner. Automatic frequency band selection for BCIs with ERDS difference maps. Proc. of the 5th International Brain-Computer Interface Conference, Graz, Austria, 2011. 